\title{
Programação em Blocos Aplicada no Ensino do Pensamento Computacional: Um Mapeamento Sistemático
}

\author{
Lucas de Lima Sousa $^{1}$, Éder Jacques Porfírio Farias ${ }^{1}$, Windson Viana de Carvalho ${ }^{2}$ \\ ${ }^{1}$ Universidade Estadual Vale do Acaraú (UVA) - Sobral, CE - Brasil \\ ${ }^{2}$ Universidade Federal do Ceará (UFC) - Fortaleza, CE - Brasil \\ lucaslima211996@gmail.com, eder_porfirioduvanet.br, windcarvalho@gmail.com
}

\begin{abstract}
In recent years, Computational Thinking (CT) has been gaining prominence on the world stage, and many researches point to the block programming paradigm as one of the main alternatives for teaching PC to people who have no programming experience. In this sense, this work proposes the realization of a Systematic Mapping Studies (SMS) on the use of block programming in the teaching of CT. During the SMS some important information was revealed, such as: which stages of formal education are studies most applied to, which are the most used tools, which types of assessment are adopted and in which countries this type of study is focused on.
\end{abstract}

Resumo. Nos últimos anos, o Pensamento Computacional (PC) vem ganhando destaque no cenário mundial e, muitas pesquisas apontam o paradigma de Programação em Blocos (PB) como uma das principais alternativas de ensino do PC para pessoas que não tenham experiência com programação. Nesse sentido, este trabalho propõe a realização de uma Mapeamento Sistemático da Literatura (MSL) sobre o uso de PB no ensino do PC. No decorrer do MSL foram reveladas algumas informações importantes, como: em quais etapas da educação formal os estudos são mais aplicados, quais são as ferramentas mais usadas, quais os tipos de avaliação adotadas e em quais países esse tipo de estudo se concentra.

\section{Introdução}

Atualmente a computação está presente em todos os lugares. Nesse contexto, não é mais possível esperar que os jovens tenham contato com conceitos relacionados a esse tema apenas quando entrarem na universidade [Barr and Stephenson 2011]. Os jovens de hoje irão se deparar com empregos que nem sequer existem ainda, e, provavelmente muitos deles estarão relacionados, direta ou indiretamente, com a computação [Barr and Stephenson 2011] [Scaico et al. 2013].

Alguns conceitos relacionados a computação, embora tenham importância social, ainda são vistos como monótonos pelos alunos. [Scaico et al. 2013] observa que muitos estudos apontam que o desinteresse por temas relacionados a essa área é causado, inúmeras vezes, devido a percepção que diversos alunos tem a respeito do assunto, considerando-o chato e entediante. Como consequência da falta de interesse, poucos alunos ingressam em cursos relacionados com computação, e com isso, a necessidade por profissionais vem aumentando. Segundo o relatório técnico apresentado por 
[SOFTEX 2013], o mercado de TI vai apresentar um deficit de cerca de 408 mil profissionais em 2022 no Brasil. Tal fato, traz a necessidade de ensinar conceitos do Pensamento Computacional (PC) cada vez mais cedo, além da necessidade de que novas metodologias de ensino do PC sejam propostas. Somando a isso, muitos estudos indicam que as diversas áreas do conhecimento estão sofrendo, direta ou indiretamente, influência do PC [Wing 2006][Bundy 2007][Barr and Stephenson 2011] [SBC 2018].

Atualmente, a utilização do paradigma de Programação em Blocos (PB), vem apresentando resultados positivos no ensino do PC. Esse paradigma vem sendo adotado por várias metodologias, desde programação de histórias, jogos, vídeos até a programação de robôs. [Weintrop et al. 2017] [Kelleher and Pausch 2005].

Visto isso, este trabalho tem como objetivo geral fazer um levantamento do uso da Programação em Blocos (PB) juntamente com o ensino do Pensamento Computacional (PC). Para tentar responder a essa questão, um Mapeamento Sistemático da Literatura (MSL) foi realizado com o propósito de conhecer e classificar as informações existentes, a fim de fornecer dados relevantes que possam embasar novas pesquisas sobre o assunto.

O restante desse trabalho está dividido da seguinte forma: Na seção 2 é apresentado um referencial teórico; A seção 3 revela a metodologia utilizada no desenvolvimento do trabalho; Na seção 4 são apresentados os resultados do trabalho, com foco em revelar as respostas para cada questão de pesquisa; Por fim, a seção 6 apresenta as considerações finais do estudo, mostrando suas limitações e trabalhos futuros.

\section{Referencial Teórico}

\subsection{Pensamento Computacional}

Segundo, [Wing 2006] "O PC envolve resolver problemas, projetar sistemas e entender o comportamento humano, baseando-se nos conceitos fundamentais da Ciência da Computação". Em um outro artigo, a autora enfatiza também que a essência do PC é a abstração, e enxerga que ela é uma ferramenta mental da computação [Wing 2008].

PC é mais que um conhecimento, ele pode ser definido como uma competência. Aprender esse conceito requer que o estudante adquira, além de um conjunto de conhecimentos, desenvolva habilidades e internalize atitudes [Wang et al. 2017]. O processo de desenvolvimento dessa competência pode ser melhorado com a utilização de novas metodologias de ensino em combinação com ferramentas computacionais. Neste contexto, o PC, pode ser entendido como uma abordagem prática na resolução de problemas, habilidade de projetar sistemas e entender a relação entre o pensamento humano e os conceitos que fundamentam a Ciência da Computação [Román-González et al. 2017].

\subsection{Programação em Blocos}

Conforme [Kelleher and Pausch 2005], a programação baseada em blocos (PB) procura abstrair a sintaxe de forma que os estudantes iniciantes mantenham seu foco apenas na semântica. Por isso, esse paradigma é um meio eficiente de atrair estudantes para o aprendizado do PC [Weintrop et al. 2017].

A PB utiliza ações de arrastar e soltar blocos gráficos ou físicos, que correspondem aos componentes de um programa escrito em linguagem textual. Cada bloco tem encaixes específicos, possibilitando que o usuário consiga visualizar onde cada um pode 
ser encaixado. Com isso, programas com sintaxes incorretas se tornam quase impossíveis, embora em alguns caso (a depender da ferramenta) seja possível encaixar blocos de forma errônea [Weintrop et al. 2017] [Kelleher and Pausch 2005].

\section{Metodologia}

O presente estudo trata-se de um MSL e tem a finalidade de fazer um levantamento do uso da PB no ensino do PC. Este trabalho procura seguir as diretrizes propostas em [Kitchenham and Charters 2007].

Como suporte para a realização do mapeamento, foi utilizada a ferramenta StArt (State of the Art through Systematic Review), software desenvolvido na Universidade Federal de São Carlos (UFSCar).

\subsection{Protocolo de Revisão}

Um protocolo de revisão indica quais os passos serão seguidos para a realização do mapeamento. A criação desse documento se faz necessária para reduzir a possibilidade de viés por parte dos pesquisadores [Kitchenham and Charters 2007].

O problema de pesquisa desse trabalho é: Entender os diversos cenários em que a Programação em Blocos está sendo usada para auxiliar o ensino e a aprendizagem do Pensamento Computacional.

As questões de pesquisa propostas por este trabalho são:

QP1. Em quais etapas da educação formal os estudos se concentram?

QP2. Quais as ferramentas utilizadas no ensino do Pensamento Computacional que fazem uso de Programação em Blocos?

QP3. Quais os tipos de avaliações de aprendizado foram empregadas nos estudos?

QP4. Em quais países os estudos se concentram?

\subsubsection{Estratégia de Busca}

Foi utilizada a busca automatizada nas seguintes bibliotecas digitais: Institute of Electrical and Electronics Engineers (IEEE), Web of Science, Association For Computing Machinery (ACM), Scopus e Science Direct. Apenas artigos escritos na língua inglesa e que foram publicados no período de 2015 a 2019, foram aceitos.

A string de busca foi composta seguindo o protocolo PICOC [Kitchenham and Charters 2007], que divide os termos em cinco blocos distintos: Population (População), Intervention (Intervenção), Comparison (Comparação), Outcome (Resultados) e Context (Contexto).

Como resultado, obteve-se o seguinte termo de busca: ("Block language" OR "Block programming" OR "Block-based programming") AND ("Computational thinking”). 


\subsubsection{Seleção dos Estudos e Extração dos Dados}

Foram propostos critérios de inclusão, exclusão e o formulário de extração dos dados. Todos descritos no protocolo de mapeamento sistemático, que foi criado antes da execução desse estudo com o intuito de sistematizar todo o processo e reduzir o viés. Os critérios de inclusão, exclusão e o formulário de extração dos dados podem ser encontrados em http: / / bit. Iy/pc_pb.

\subsection{Aplicação do Protocolo}

A Figura 1 ilustra o processo de busca dos estudos, seleção dos trabalhos e extração dos dados. Esse processo é dividido em 5 fases principais: escolha das bases de dados online; criação da string de busca; coleta dos estudos; seleção dos estudos e extração dos dados. As fases secundárias também estão expressas na Figura 1.

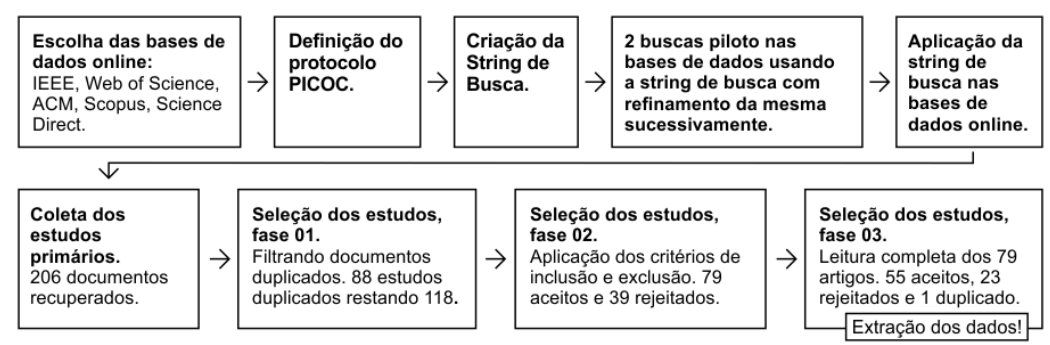

Figura 1. Representação da estratégia de busca e seleção dos estudos primários.

A string de busca foi executada e o resultado obtido está descrito na tabela 1. Nela é possível observar que 206 estudos foram recuperados utilizando a string de busca nas bibliotecas online. A base de dados Scopus apresentou a maior concentração de estudos, sendo responsável por 35,4\% de todos os estudos recuperados. Em contrapartida, a IEEE foi a que menos ofereceu trabalhos, tendo apenas 11,2\% de artigos. Depois que o conjunto com 206 estudos primários foi recuperado, eles passaram por três fases de seleção.

Tabela 1. Estudos recuperados na busca automatizada

\begin{tabular}{|l|c|c|}
\hline Base de dados & Quantidade de estudos & \% \\
\hline IEEE & 23 & $11,2 \%$ \\
\hline Web of Science & 45 & $21,8 \%$ \\
\hline ACM & 38 & $18,4 \%$ \\
\hline Scopus & 73 & $35,4 \%$ \\
\hline Science Direct & 27 & $13,1 \%$ \\
\hline Total: & $\mathbf{2 0 6}$ & $\mathbf{1 0 0 \%}$ \\
\hline
\end{tabular}

Na primeira fase, os estudos duplicados foram detectados com auxílio da ferramenta StArt e excluídos. No total, 88 estudos foram identificados como duplicados sobrando 118 trabalhos. Na segunda fase, os títulos, palavras-chave e resumos de todos os trabalhos restantes foram lidos e julgados segundo os critérios de inclusão e exclusão. Nessa fase, 39 estudos foram rejeitados e 79 foram aceitos. Na terceira e última fase foi aplicado novamente os critérios de seleção, mas dessa vez com a leitura completa dos 79 artigos retantes. Nessa etapa 55 estudos foram aceitos, 23 rejeitados e 1 foi identificado 
como duplicado. Ainda na terceira fase de seleção, os dados necessários para responder as questões de pesquisa foram extraídos com auxílio do formulário de extração. A planilha contendo todos os 55 trabalhos selecionados e os 62 rejeitados pode ser acessada através do link http://bit.1y/pc_pb.

\section{Resultados e Discussões}

Nesta seção, os dados obtidos no MSL são sintetizados e analisados com foco em responder as questões de pesquisa previamente estabelecidas. Além disso, foi possível extrair algumas informações adicionais.

Pode ser observado na Figura 2, que no período estabelecido para realização desse MSL (2015 a 2019), houve um crescimento de publicações que relacionam PB no ensino do PC. Com mais trabalhos sendo publicados, é esperado que o ensino de PC utilizando $\mathrm{PB}$, se torne uma prática cada vez mais comum.

\section{Figura 2. Ano de publicação dos estudos primários}

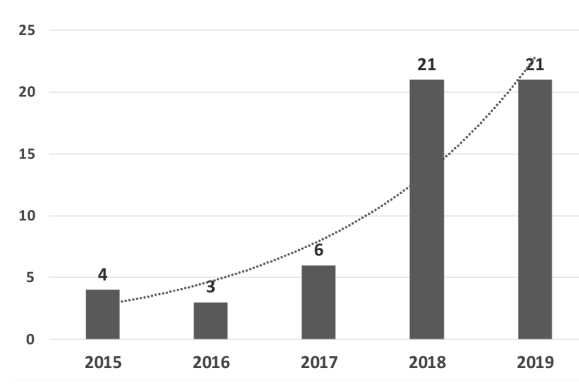

\subsection{Respostas às Questões de Pesquisa}

Esta seção apresenta as respostas para as questões de pesquisa, com base na análise dos dados extraídos dos estudos primários selecionados.

\section{QP1- Em quais etapas da educação formal os estudos se concentram?}

A Figura 3 apresenta a quantidade de estudos por etapa da educação formal. Como forma de normalizar todas as etapas, foi utilizado o padrão adotado no Brasil (pré-escola, ensino fundamental, ensino médio e ensino superior), uma vez que os países adotam classificações diferentes para as etapas educacionais. É importante dizer que vários estudos tem seu foco em mais de uma etapa educacional. Dessa forma, alguns estudos foram vinculados a mais de uma categoria.

Analisando o gráfico, pode-se notar que a maioria dos estudos está concentrado no ensino fundamental e médio, o que era esperado, pois são nessas etapas da educação que o uso de PB é recomendado. A categoria 'Não se aplica' enumera os trabalhos que não vincularam seus estudos a nenhuma etapa educacional.

QP2- Quais as ferramentas utilizadas no ensino do Pensamento Computacional que utilizam Programação em Blocos?

Como mostrado na Figura 4, foi detectado um grande número de ferramentas que usam o paradigma de PB. No total, foram 33 ferramentas diferentes e um trabalho que não identificou a ferramenta usada. A ferramenta mais utilizada foi o Scratch, sendo incluída 
IX Congresso Brasileiro de Informática na Educação (CBIE 2020)

Anais do XXXI Simpósio Brasileiro de Informática na Educação (SBIE 2020)

Figura 3. Etapas da educação formal onde os estudos se concentram

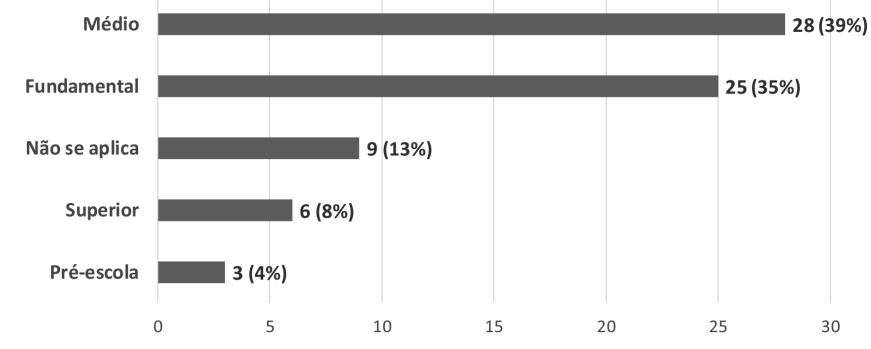

em 23 dos trabalhos analisados, o que resultou em um percentual de $30 \%$. Na sequência, o App inventor e o Pencil Code ficaram em segundo e terceiro lugar, respectivamente. O restante das ferramentas apareceram em apenas um ou dois trabalhos, o que demonstra uma grande pulverização no uso de ferramentas. A categoria "outros" agrupa as 24 ferramentas que apareceram em apenas um trabalho. O gráfico completo com todas as ferramentas pode ser encontrado em http: / / bit. ly / pc_pb.

Outra observação interessante é que $59 \%$ das ferramentas são aplicações web. Esse resultado é apresentado na Figura 5, e pode ser justificado pelo fato de que essas ferramentas possuem maior portabilidade e por serem de fácil distribuição.

Figura 4. Ferramentas de programação em blocos

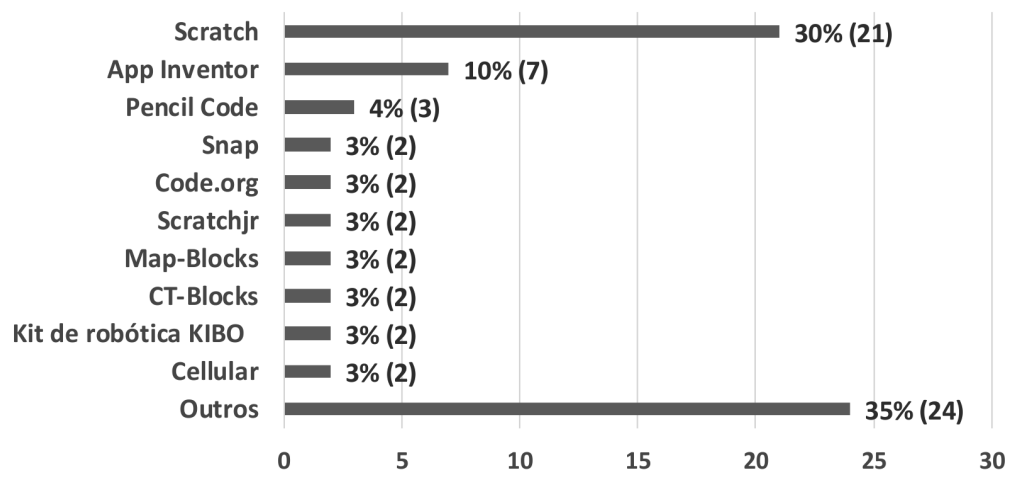

Figura 5. Plataformas onde as ferramentas são executadas

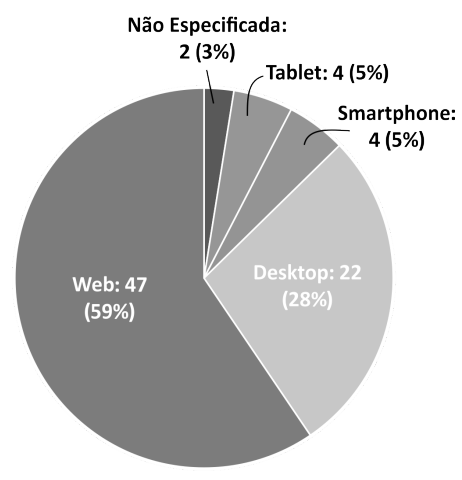
estudos?

QP3- Quais os tipos de avaliações de aprendizado que foram empregadas nos 
De forma geral, os trabalhos apresentaram diversos tipos de avaliações. Devido essa granularidade, as avaliações foram distribuídas em categorias para que os dados pudessem ser agrupados e assim melhor representados.

A Figura 6 mostra a distribuição dos trabalhos de acordo com as categorias propostas. A categoria "Teste avaliativo" reúne o conjunto de estudos que utilizaram testes de conhecimento comumente aplicados em salas de aula, como testes escritos, práticos e análise de portfólio, que corresponde a $20 \%$ das avaliações mapeadas. A avaliação por "Observação empírica" contém o conjunto de trabalhos que procuraram avaliar os alunos por meio de monitoramento de atividades e esteve presente em $16 \%$ dos tipos de avaliações. Logo em seguida com 14\% , a categoria "Teste automatizado" agrupa os trabalhos que fizeram uso de ferramentas digitais para medir o nível de PC e outras habilidades dos alunos, como por exemplo, o grau de engajamento nas atividades. As demais categorias propostas foram: "Pesquisa", "Rubrica", "Entrevista" e "Gravação", que aparecerem em 10\%, 10\%, 6\% e 5\% das avaliações, respectivamente. A categoria "Não se aplica" apresenta os trabalhos que não fizeram nenhum tipo de avaliação do conhecimento e corresponde a 19\% dos trabalhos, o que demonstra que um número muito grande de trabalhos da área não fazem avaliação dos resultados. É preciso deixar claro que um mesmo trabalho pode ter utilizado diferentes formas de avaliação, por isso alguns estudos estão distribuídos em mais de uma categoria.

\section{Figura 6. Tipos de avaliações empregadas}

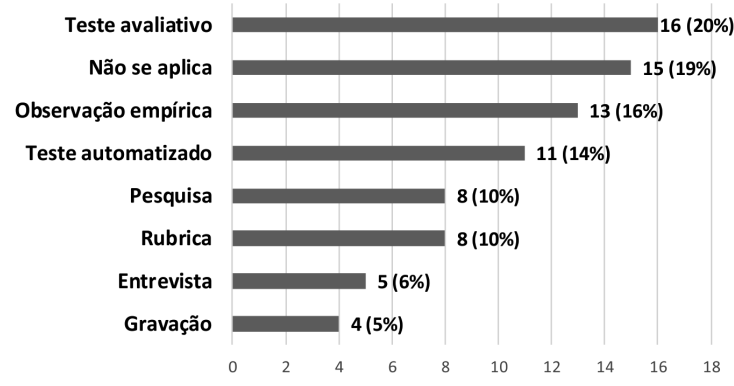

Para um melhor entendimento de como se deu a categorização dos trabalhos, a tabela 2 apresenta uma visão geral das subcategorias de avaliações constantes em cada uma das categorias descritas.

\section{QP4- Em quais países os estudos se concentram?}

Neste MSL, um total de 19 países apresentam publicações sobre o uso de PB no ensino do PC. Como demonstrado na Figura 7, o país com maior concentração de estudos sobre o assunto é os Estados Unidos, com 20 documentos selecionados para esse MSL, o que contabiliza 34,5\%, seguido da Índia com 8 (13,8\%) e logo após a Coreia do Sul com 6 trabalhos selecionados (10,3\%). Apenas 3,4\% dos trabalhos foram realizados no Brasil. A categoria "Outros" corresponde a um total de 10 países que tiveram 1 artigo cada. O gráfico completo com todos os países pode ser encontrado em http://bit. $I_{\mathrm{y}} / \mathrm{pc}$ $\mathrm{pb}$.

É válido salientar que, 3 artigos foram publicados descrevendo dois países ao mesmo tempo. Esses artigos foram contabilizados como sendo um para cada país, dessa forma o somatório total foi 58 e não 55 trabalhos. 
IX Congresso Brasileiro de Informática na Educação (CBIE 2020)

Anais do XXXI Simpósio Brasileiro de Informática na Educação (SBIE 2020)

Tabela 2. Categorias e Subcategorias dos tipos de avaliação

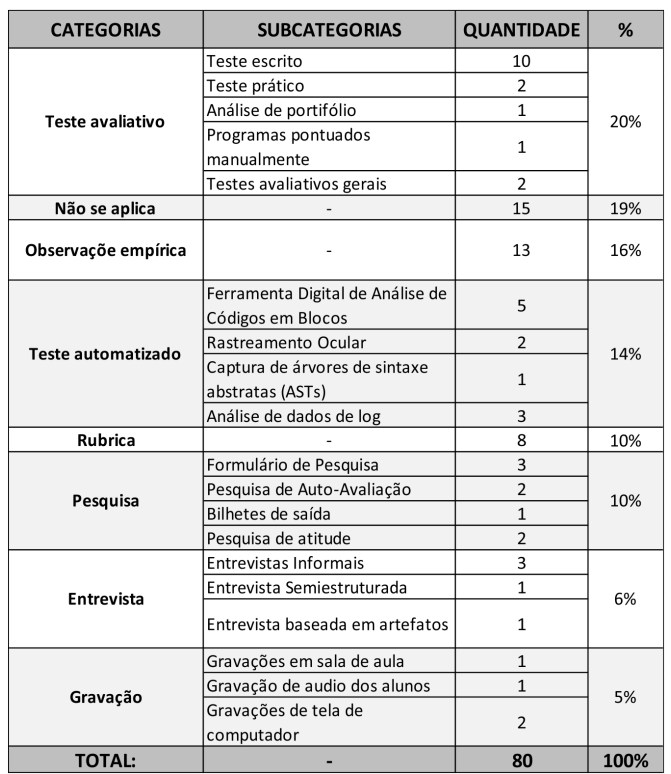

Figura 7. Países onde os estudos se concentram

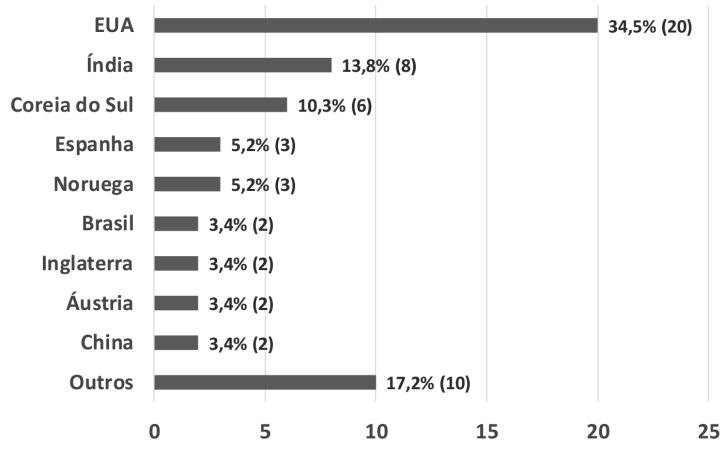

\section{Trabalhos Relacionados}

Existem alguns trabalhos que fizeram uso de um MSL a fim de levantar informações sobre PC. [Alves et al. 2019] realizou um MSL para levantar quais são as avaliações usadas para avaliar programas de computadores construídos através de PB, no contexto do ensino fundamental e médio. Já [Santos et al. 2018] realizou um MSL (tendo como foco o cenário brasileiro) a fim de levantar informações sobre a diversidade de experiências que tratam de pensamento e programação computacional na educação básica. [Martins-Pacheco et al. 2019] fez uso de um MSL para levantar informações sobre o que está sendo feito para avaliar o PC no contexto da educação básica.

Este MSL se diferencia dos trabalhos citados pois procura fazer um levantamento de estudos que utilizam PB, juntamente com o ensino do PC no cenário mundial e em todas as fases do ensino formal.

\section{Considerações Finais}

Esta seção apresentará as conclusões extraídas no decorrer deste trabalho, destacando os principais resultados obtidos e propondo alguns trabalhos futuros. 
Pode-se perceber que está havendo um crescimento nas publicações a respeito do uso de PB no ensino do PC. Tal tendência é um forte indício do crescimento da área estudada.

Outro resultado interessante, é o grande número de ferramentas utilizadas, e apesar de o Scratch ter aparecido em um número considerável de estudos (30\%), a grande quantidade de ferramentas existentes corrobora com o indicativo de que a área esteja em pleno crescimento. O fato de que quase $60 \%$ das ferramentas utilizadas serem web demonstra que a área segue uma tendência global, que é a substituição de ferramentas desktop por ferramentas $w e b$.

No mapeamento dos tipos de avaliação utilizados, pôde-se perceber que são utilizadas várias formas de avaliação diferentes com uma distribuição quase uniforme, como um leve protagonismo para os testes avaliativos. Contudo, o que chama atenção é que quase $20 \%$ dos artigos não fizeram uso, ou pelo menos não informaram, de nenhuma ferramenta de avaliação, o que pode indicar a necessidade de um maior rigor científico para os estudos na área.

Por fim, é válido salientar que a grande maioria dos estudos que se apresentaram para este MSL foram realizados nos Estados Unidos e que apenas 3,4\% deles foram feitos no Brasil, indicando que ainda é uma área com forte potencial de estudo em nosso país. É claro que esse resultado deve ser analisado conforme as restrições do MSL, uma vez que apenas estudos escritos na língua inglesa foram selecionado e esse fator pode ter contribuído para justificar essa quantidade.

A pesquisa teve como objetivo geral fazer um levantamento do uso de PB, juntamente com o desenvolvimento do PC. Constatou-se que o objetivo geral foi atendido com sucesso.

Embora este trabalho procure seguir um protocolo pré-estabelecido a fim de fornecer resultados confiáveis, algumas ameaças a validade podem ser apresentadas. Entre elas, pode-se destacar o fato de que o MSL é feito com base na análise dos pesquisadores, e pode, de alguma forma, introduzir análises enviesadas, mesmo que involuntárias. A string de busca também é um fator limitante, pois alguns trabalhos sobre o tema podem não ter sido recuperados e com isso não foram ser selecionados para este MLS.

Como trabalhos futuros pretende-se explorar melhor, realizando uma revisão sistemática de literatura, a utilização de plataformas web que buscam desenvolver o PC usando PB. Visto que foi observado uma grande adesão por tais sistemas.

Pretende-se também realizar um estudo com foco nos testes automatizados que medem o nível de PC. Uma vez que haja adesão do ensino de PC no contexto da escola básica é esperado que os professores nesse cenário não possuam habilidades suficiente para transmitir aos alunos. Testes automatizados podem ser bem úteis nesse processo inicial.

\section{Referências}

Alves, N. D. C., Von Wangenheim, C. G., and Hauck, J. C. (2019). Approaches to assess computational thinking competences based on code analysis in K-12 education: A systematic mapping study. Informatics in Education, 18(1):17. Publisher: Institute of Mathematics and Informatics. 
IX Congresso Brasileiro de Informática na Educação (CBIE 2020)

Anais do XXXI Simpósio Brasileiro de Informática na Educação (SBIE 2020)

Barr, V. and Stephenson, C. (2011). Bringing computational thinking to K-12: what is Involved and what is the role of the computer science education community? Acm Inroads, 2(1):48-54. Publisher: ACM New York, NY, USA.

Bundy, A. (2007). Computational thinking is pervasive. Journal of Scientific and Practical Computing, 1(2):67-69.

Kelleher, C. and Pausch, R. (2005). Lowering the barriers to programming: A taxonomy of programming environments and languages for novice programmers. ACM Cотриting Surveys (CSUR), 37(2):83-137. Publisher: ACM New York, NY, USA.

Kitchenham, B. and Charters, S. (2007). Guidelines for performing systematic literature reviews in software engineering.

Martins-Pacheco, L. H., von Wangenheim, C. A. G., and da Cruz Alves, N. (2019). Assessment of Computational Thinking in K-12 Context: Educational Practices, Limits and Possibilities-A Systematic Mapping Study. In Proceedings of the 11th International Conference on Computer Supported Education (CSEDU 2019), volume 1, pages 292-303.

Román-González, M., Pérez-González, J.-C., and Jiménez-Fernández, C. (2017). Which cognitive abilities underlie computational thinking? criterion validity of the computational thinking test. Computers in Human Behavior, 72:678-691.

Santos, P. S., Araujo, L. G. J., and Bittencourt, R. A. (2018). A mapping study of computational thinking and programming in brazilian k-12 education. In 2018 IEEE Frontiers in Education Conference (FIE), pages 1-8. IEEE.

SBC (2018). Manifesto da SBC pela Inserção de Computação na Educação Básica. Library Catalog: www.sbc.org.br.

Scaico, P. D., de Lima, A. A., Azevedo, S., da Silva, J. B. B., Raposo, E. H., Alencar, Y., Mendes, J. P., and Scaico, A. (2013). Ensino de programação no ensino médio: Uma abordagem orientada ao design com a linguagem scratch. Revista Brasileira de Informática na Educação, 21(02):92.

SOFTEX (2013). Mercado de trabalho e formação de mão de obra em ti. Cadernos Temáticos do Observatório.

Wang, L., Sy, A., Liu, L., and Piech, C. (2017). Learning to represent student knowledge on programming exercises using deep learning. International Educational Data Mining Society.

Weintrop, D., Shepherd, D. C., Francis, P., and Franklin, D. (2017). Blockly goes to work: Block-based programming for industrial robots. In 2017 IEEE Blocks and Beyond Workshop (B B), pages 29-36.

Wing, J. M. (2006). Computational thinking. Commun. ACM, 49(3):33-35.

Wing, J. M. (2008). Computational thinking and thinking about computing. Philosophical Transactions of the Royal Society A: Mathematical, Physical and Engineering Sciences, 366(1881):3717-3725. Publisher: The Royal Society London. 